\title{
B-cell-receptor-targeted therapy in CLL
}

\section{Dr. Haitham Abdelbary, MD}

\section{Lecturer of Internal Medicine, Department of Hematology And Bone Marrow Transplantation, Ain Shams University}

\section{Introduction}

Chronic lymphocytic leukemia (CLL) occurs most frequently in patients age $>70$ years and is similar genetically to small lymphocytic lymphoma (SLL), where blood lymphocytosis is lacking. The natural history of CLL progression is variable and influenced in great part by genetic, epigenetic, and biochemical properties of the tumor cells and clinical features at time of diagnosis.

On the basis of earlier studies demonstrating no benefit of early treatment with alkylator-based therapy, treatment of CLL is not recommended until symptoms develop. However, during the past 5 years, the application of genomic studies and introduction of many new therapies for CLL have greatly increased the complexity of treating symptomatic CLL. In addition, new targeted therapy offers the possibility of a paradigm shift in this disease ${ }^{1}$.

There is now strong evidence that signaling via the B-cell receptor (BCR) plays a major role in the development of CLL and that it determines the variable clinical behavior. In this Perspective, we discuss the functional significance of the BCR in CLL and we describe strategies to target BCR signaling as a new therapeutic approach ${ }^{2}$.

\section{BCR-mediated signaling in normal B cells}

\section{Signalosome activation}

For a consideration of signaling in CLL, it is first useful to recap consequences of slg engagement in normal B cells. Initial antigen binding leads to the formation of the "signalosome," a complex of kinases and scaffold proteins tethered at the plasma membrane at sites of slg activation (Figure 1A). The "trigger" event in the formation of the signalosome is phosphorylation of the immunoreceptor tyrosine-based activation motifs in the C-terminal tail of BCR-associated Iga (CD79A) and $\lg \beta$ (CD79B) by the SRC-family kinase LYN.

Phosphorylated immunoreceptor tyrosine based activation motifs act as docking sites to recruit the tyrosine kinase SYK through its tandem SH2 domain, leading to SYK activation via SRC-family kinase-dependent phosphorylation and autophosphorylation. 
The BCR signal is further propagated by SYK via association with the adaptor molecule B-cell linker protein (BLNK) and its downstream signaling components Bruton tyrosine kinase (BTK) and phospholipase CY2 (PLC-Y2). LYN dependent phosphorylation of the cytoplasmic domain of CD19 also recruits the p85 subunit of phosphoinositide 3-kinase $(\mathrm{PI} 3 \mathrm{~K})^{3}$.

\section{Downstream signaling}

After formation of the signalosome, the second phase of BCR signaling involves the activation of distal signaling molecules (Figure 1B). Activation of PLC-y2 leads to the generation of the downstream second messengers inositol-1,4,5-triphosphate and diacylglycerol, which induce the release of intracellular $\mathrm{Ca}^{2}+$ and activate protein kinase C, respectively.

Protein kinase $\mathrm{C}$ subsequently induces the activation of transcription factors, including NF- $\mathrm{KB}$ and nuclear factor of activated T cells. Recruitment of PI3K to the plasma membrane leads to the production of phosphatidylinositol 3,4,5-triphosphate, which is required for optimal activation of BTK, as well as for recruitment of 3'-phosphoinositide-dependent kinase and subsequent activation of AKT.

PLC-y2 is also involved in the activation of mitogen-activated protein kinase pathways, including the extracellular signal-regulated kinase 1/2 (ERK1/2), c-Jun NH2-terminal kinase, and p38 kinases (Figure 1B). The ERK/mitogen activated protein kinase pathway can also be regulated by RAS/RAF1 signaling ${ }^{4}$.

The third phase of events involves modulation of multiple downstream regulators, which ultimately mediate changes in cell proliferation, survival, and migration, via both transcriptional modulation and phosphorylation (Figure 1B). For example, BCR signaling leads to modulation of key regulators of cell survival (eg, MCL1, BIM) and cell cycle (cyclin D2, MYC).

\section{Negative regulation}

Negative regulators of BCR signalosome signaling, such as CD22, CD5, CD72, and FcyRIIB, are essential in controlling the duration and intensity of the BCR signal (Figure 1A).

These receptors contain immunoreceptor tyrosine-based inhibition motifs, which are phosphorylated by LYN on BCR stimulation. This leads to the recruitment of inhibitory phosphatases, such as $\mathrm{SH} 2$ domain containing tyrosine phosphatase-1, $\mathrm{SH} 2$ domain-containing phosphatidyl 5-phosphatase-1 and -2 , and protein tyrosine 
phosphatase nonreceptor type 22, which attenuate BCR signaling. Therefore, LYN both positively and negatively regulates signal transduction via the $\mathrm{BCR}^{5}$.

\section{BCR-mediated signaling in CLL cells}

\section{Heterogeneity of responses}

BCR signaling responses in CLL cells are heterogeneous. At one extreme is the subset of CLL samples that appear to be essentially unresponsive to slgM engagement, at least using anti-lgM as a surrogate for antigen, this is most common among M-CLL and is associated with indolent disease ${ }^{6}$.

However, even in the subset of samples that do retain slgM signaling responsiveness, responses in vitro are variable between cases and partial, with effective activation of only some downstream responses. For example, despite activation of ERK1/2, there is weak activation of p38 and c-Jun $\mathrm{NH} 2$-terminal kinase ${ }^{7}$. The activation of nuclear factor- $\mathrm{kB}$ is also variable compared with ERK $1 / 2^{7}$.

The levels of slgM appear to be one major determinant of these heterogeneous signaling responses. slgM levels are generally but variably reduced in CLL compared with normal naive $B$ cells. This may be at least partly a consequence of repeated antigen engagement and receptor down-modulation. Strong down-modulation appears to contribute to lack of slgM signaling responses, especially in ${\mathrm{M}-\mathrm{CLL}^{6}}^{6}$.

\section{Modulation of slgM-mediated signaling}

Although slgM modulation plays an important role in determine signaling response, other factors must also contribute. Expression of the ZAP70 tyrosine kinases identifies CLL patients with a more aggressive disease and earlier time to treatment ${ }^{8}$.

Interestingly, BCR signaling capacity in CLL correlates with ZAP70 expression and signaling can be augmented by ZAP70 overexpression ${ }^{9}$. However, the effect of ZAP70 is independent of its kinase activity. ZAP70 may indirectly enhance SYK activation and/or sequester SYK inhibitors ${ }^{10}$. There is also evidence that ZAP70 may modulate other signaling pathways, in particular those contributing to cell migration.

Expression of CD38 expression is also a prognostic indicator and expression correlates with slgM signaling capacity. CD38 associates with slgM in CLL cells, but it is unclear whether CD38 can directly modulate signaling responses ${ }^{11}$.

Interestingly, CD38-CD31 interactions also appear to contribute to pathways involved in migration and homing and enhance CLL survival via induction of BCL2 and BCL-XL. 


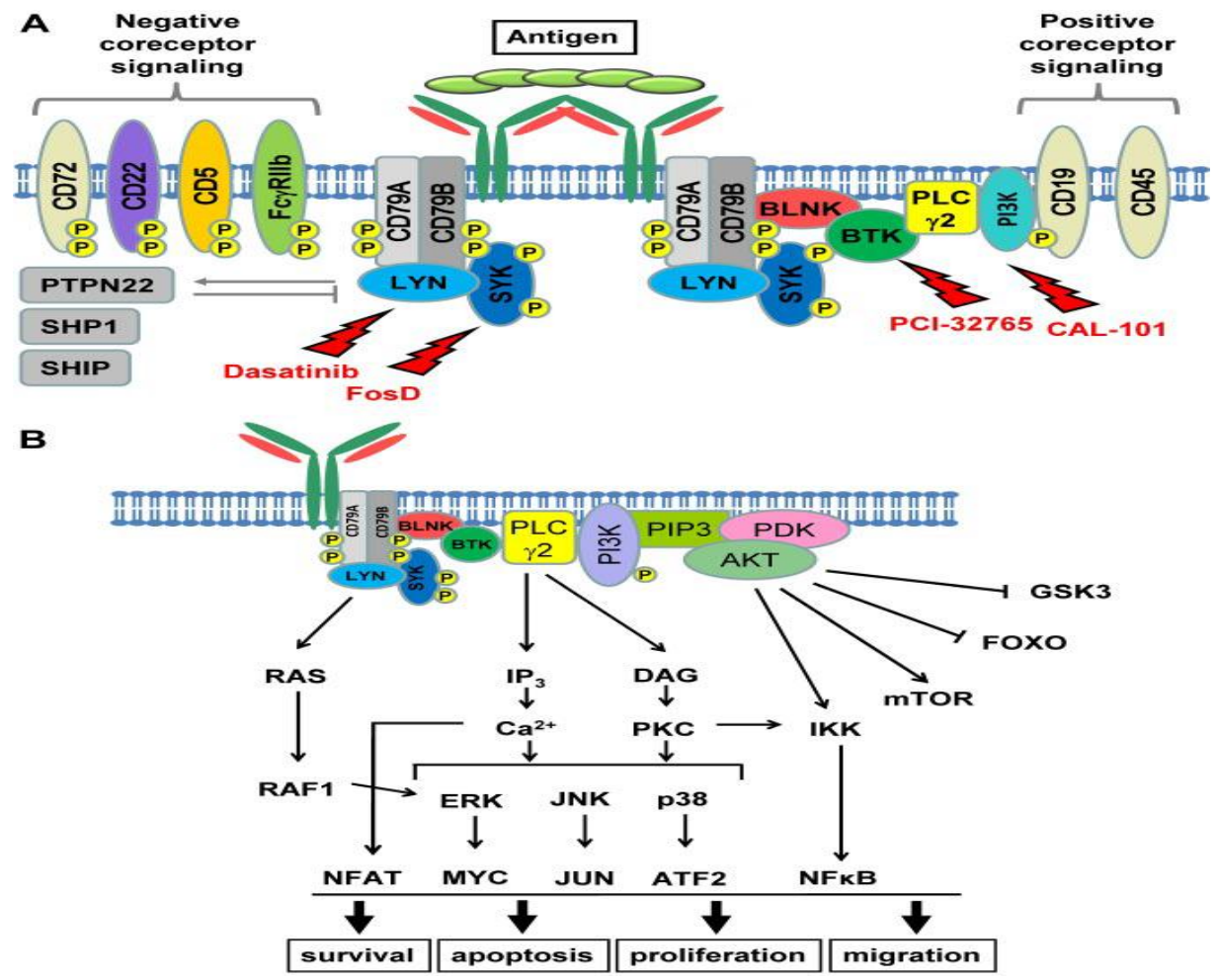

Figure 1. B-cell receptor signaling in CLL and targeted inhibition.

The diagram shows the major signaling pathways activated after BCR activation. (A) The signalosome is composed of LYN, SYK, BLNK, BTK, PLC-Y2, and PI3K. Chemical inhibition of LYN, SYK, BTK, and PI3K by dasatinib, fostamatinib disodium (FosD), PCl- 32765 (ibrutinib), or CAL-101 (idelalisib), respectively, blocks BCR signaling in CLL cells in vitro. The BCR signal can be further enhanced or inhibited by positive and negative coreceptor signaling to control the duration and intensity of the signal. (B) Principal downstream signaling pathways linking the BCR to biologic responses

\section{B-cell-receptor-targeted therapy in treatment of relapsed CLL lbrutinib}

The first BTK inhibitor to enter the clinic was the orally bioavailable, irreversibly binding small molecule ibrutinib (PCI-32765). 
Ibrutinib has shown exceptional activity in relapsed CLL. A phase I study of ibrutinib in relapsed B-cell malignancies was initiated, where durable clinical activity was noted in nine of 16 patients with CLL or SLL ${ }^{12}$.

Notable among these patients with CLL receiving ibrutinib was early lymphocytosis, which now is recognized to be a class effect of all BCR antagonists. This occurs early with therapy but is otherwise accompanied by reduction in organomegaly, lymph node size, and cytopenias, thereby differentiating it from typical progression seen with chemoimmunotherapy.

No dose-limiting toxicity was identified with ibrutinib at any dose. This study was followed by a phase IB/II study of ibrutinib, where 85 patients with relapsed or refractory CLL or SLL were treated at two different doses (420 and $840 \mathrm{mg}$ daily) ${ }^{13}$. This group was heavily pretreated (median, four prior therapies) and had a high proportion of patients with advanced-stage disease and del (17) (p13.1).

The ORR by IWCLL 2008 criteria 57 was 71\% (CRs, two; PRs, 34) in the 420-mg cohort and $71 \%$ (PRs, 24) in the 840-mg cohort. In addition, 10 (20\%) and five patients (15\%) in the 420- and 840-mg cohorts, respectively, demonstrated a nodal response with persistent lymphocytosis.

Response to ibrutinib did not vary based on cytogenetic adverse features previously identified in CLL. Most notably, of the 28 patients with del(17)(p13.1) enrolled onto this study, $18(68 \%)$ responded. The 26-month estimated PFS rate for all patients enrolled onto this study was $75 \%$. Unlike response, PFS did differ by genomic group; 26 month estimated PFS rate was 56\% when either del (11) (q22.3) or del (17) (p13.1).

Extended therapy with ibrutinib was well tolerated in this trial, with common adverse events including grade 1 to 2 diarrhea, cough, fatigue, upper respiratory infections, nausea, fever, peripheral edema, myalgias, and petechiae or ecchymoses. Although grade $>3$ infections occurred frequently in this study, the occurrence decreased with time, where the average rate per 100 patient-months within the first 6 months was 7.1 but 2.6 thereafter. Most problematic was the low frequency of subarachnoid hemorrhages in several patients receiving concomitant warfarin treatment but no other anticoagulants or antiplatelet agents. This unusual, atypical CLL toxicity prompted prohibition of concurrent therapy with oral vitamin $\mathrm{K}$ antagonists in all subsequent studies of ibrutinib. In addition, ibrutinib is typically held for 3 to 7 days before and after surgical procedures in a further attempt to avoid bleeding problems. BTK does not seem 
essential for platelet activation, but a role for BTK inhibition in stable thrombus formulation has been postulated. Studies of platelet function in patients with BTKinactivating mutations have demonstrated abnormalities of collagen- and collagenrelated peptide-induced aggregation but not bleeding ${ }^{14}$.

These results demonstrating durable remissions prompted initiation of a large randomized phase III study (RESONATE) comparing ibrutinib monotherapy with ofatumumab in patients with relapsed CLL (Table 1). This trial was recently ended by the data safety and monitoring committee because of the significant benefit seen in both PFS and OS with ibrutinib. On the basis of these collective results, ibrutinib represents the best salvage therapy for relapsed CLL irrespective of genomic risk type unless longterm anticoagulation with warfarin is required.

Idelalisib (CAL-101, GS-1101) is a first-in-class, selective oral inhibitor of the $\mathrm{p} 110 \delta$ isoform of PI3Kס. Preclinical work with this molecule demonstrated that this small molecule inhibited both intrinsic and extrinsic survival signals, including those generated by BCR signaling in CLL.

\section{Idelalisib Therapy}

The initial phase I study with idelalisib enrolled 54 patients with relapsed or refractory CLL who were treated with continuous dosing until progression ${ }^{15}$. Like with ibrutinib, early lymphocytosis was observed concomitantly with reduction in nodal size.

After a median 9 months of drug exposure, the ORR was $39 \%$, with nodal response observed in a larger proportion of patients (81\%). The median PFS was 17 months.

Dose-limiting toxicities were not observed, and potentially treatment-related adverse events (chiefly fatigue, rash, diarrhea, respiratory tract infections, and reversible elevations of hepatic transaminases) resulted in discontinuation of treatment in only $7 \%$ of patients. Subsequent combination studies with rituximab, bendamustine, BR, ofatumumab, and chlorambucil were performed, demonstrating all these approaches resulted in higher ORRs and acceptable toxicity ${ }^{16}$.

Several phase III studies were initiated, including combination approaches with BR, ofatumumab, and rituximab. The only fully published study administered rituximab with idelalisib or placebo as part of salvage therapy. This study was closed prematurely because the combination therapy demonstrated a significantly higher ORR ( $81 \% v 13 \%)$, 
PFS rate, and OS rate at 12 months (92\% $v$ 80\%). Grade 3 diarrhea, rash, and liver function abnormalities were more common with the combination therapy.

Table 1: Ongoing phase III Studies with potential to change standard of care in CLL.

\begin{tabular}{|c|c|c|c|}
\hline Study & Treatment Comparison & Treatment Status & $\begin{array}{l}\text { Age } \\
\text { Group } \\
\text { (years) }\end{array}$ \\
\hline CLL12 & Ibrutinib $v$ placebo & High risk, aymptomatic & $\geq 18$ \\
\hline RESONATE II & $\begin{array}{l}\text { CLB v ibrutinib } \\
\text { (crossover) }\end{array}$ & $\begin{array}{l}\text { Symptomatic. } \\
\text { untreated }\end{array}$ & $\geq 65$ \\
\hline A041202 & $\begin{array}{l}\text { BR } v \text { ibrutinib plus } \\
\text { rituximab } v \text { ibrutinib } \\
\text { (crossover) }\end{array}$ & $\begin{array}{l}\text { Symptomatic, } \\
\text { untreated }\end{array}$ & $\geq 65$ \\
\hline E1912 & FCR $V$ IR & $\begin{array}{l}\text { Symptomatic, } \\
\text { untreated }\end{array}$ & $<70$ \\
\hline NCT01980889 & $\begin{array}{l}\text { BR plus placebo } v \text { BR } \\
\text { plus idelalisib }\end{array}$ & $\begin{array}{l}\text { Symptomatic. } \\
\text { untreated }\end{array}$ & $\geq 18$ \\
\hline NCT01980875 & $\begin{array}{l}\text { Idelalisib plus rituximab } \\
v \text { placebo plus } \\
\text { rituximab or idelalisib } \\
\text { plus CLB } v \text { placebo } \\
\text { plus CLB }\end{array}$ & $\begin{array}{l}\text { Symptomatic. } \\
\text { untreated }\end{array}$ & $\geq 65$ \\
\hline CLLM1 & $\begin{array}{l}\text { Lenaldiomide } v \text { placebo } \\
\text { for consolidation } \\
\text { after initial therapy }\end{array}$ & $\begin{array}{l}\text { Consolidation after first } \\
\text { treatment }\end{array}$ & $\geq 18$ \\
\hline RESONATE & $\begin{array}{l}\text { Ofatumumab } v \\
\text { ibrutinib }\end{array}$ & Relapsed & $\geq 18$ \\
\hline NCT01611090 & $\begin{array}{l}\text { BR plus placebo } v \text { BR } \\
\text { plus ibrutinib }\end{array}$ & Relapsed & $\geq 18$ \\
\hline NCT01569295 & $\begin{array}{l}\text { BR plus placebo } v \text { BR } \\
\text { plus idelalisib }\end{array}$ & Relapsed & $\geq 18$ \\
\hline NCTO2005471 & $\begin{array}{l}\text { ABT199 plus rituximab } \\
\text { V BR }\end{array}$ & Relapsed & $\geq 18$ \\
\hline
\end{tabular}

Abbreviations: BR, bendamustine plus rituximab; CLB, chlorambucil; CLL, chronic lymphocytic leukemia; FCR, fludarabine, cyclophosphosphamide, and rituximab; IR, ibrutinib plus rituximab; RESONATE, A Phase III Study of lbrutinib (PCl-32765) Versus Ofatumumab in Patients With Relapsed or Refractory Chronic Lymphocytic Leukemia; RESONATE II, A Multicenter, Open-Label, Phase III Study of the Bruton's Tyrosine Kinase Inhibitor PCl32765 Versus Chlorambucil in Patients 65 Years or Older With TreatmentNaive Chronic Lymphocytic Leukemia or Small Lymphocytic Lymphoma.

Duvelisib (IPI-145) is the second PI3K-targeted agent to enter clinical development. IPI-145 potently inhibits both the p110ס and p110 $\mathrm{Y}$ isoforms of the enzyme at pico- and nanomolar concentrations, respectively.

Relapsed/refractory CLL patients (N 34; 22 evaluable for response) were treated with escalating doses from 8 to $100 \mathrm{mg}$ twice per day; at the higher dose, 2 dose-limiting toxicities were reported (grade 3 rash, grade 3 elevation of hepatic transaminases). The median time to response (1.9 months) and pattern of response (ORR, 55\%) was similar to the $\delta$-specific agent. Similar to idelalisib, the nodal response rate was impressive $(87 \%)^{17}$.

However, the addition of $\mathrm{p} 110 \mathrm{y}$ inhibition at higher doses of IPI-145 appeared to result in more significant immune suppression; Pneumocystis pneumonia has been observed, and prophylaxis for opportunistic infections will be mandated going forward. Accrual continues to expansion cohorts enrolling both relapsed/refractory and treatment-naïve disease. 
CLL is a disease in which bcl-2 is overexpressed that also appears to be influenced by BTK inhibition. ABT-199, a small molecule inhibitor of BCL2, might be rationally combined with ibrutinib in this setting, given the promising results emerging from phase 1 studies $^{18}$.

\section{Symptomatic, untreated elderly patients}

\section{lbrutinib therapy}

Limited data suggest even more impressive efficacy in previously untreated patients with CLL. A study of 31 patients age > 65 years were treated with ibrutinib $420 \mathrm{mg}$ daily until progression or unacceptable toxicity. The median age was 71 years, and more than half of patients had advanced Rai stage disease. Only $9 \%$ of patients had high-risk genomic features, either del (11) (q22.3) or del (17) (p13.1).

The ORR according to 2008 International Workshop on Chronic Lymphocytic Leukaemia (IWCLL) criteria was $71 \%$, and an additional $13 \%$ of patients achieved partial responses (PRs) with lymphocytosis. At a median follow-up of 24 months, PFS and OS rates were $96 \%$. Toxicity was similar to that observed in patients treated for relapsed disease ${ }^{19}$.

These results are quite remarkable, but they only applied to predominately low-genomic risk patients. These promising data prompted several ongoing phase III trials of ibrutinib in the elderly.

RESONATE II (A Multicenter, Open-Label, Phase III Study of the Bruton's Tyrosine Kinase Inhibitor PCl-32765 Versus Chlorambucil in Patients 65 Years or Older With Treatment-Naive Chronic Lymphocytic Leukemia or Small Lymphocytic Lymphoma).

The Alliance A041202 trial, which is actively accruing patients, will compare chemoimmunotherapy (BR) with either ibrutinib or ibrutinib plus rituximab in patients age $>65$ years and represents the first comparison of chemoimmunotherapy with ibrutinib-containing regimens in elderly patients.

\section{Ibrutinib Combination Therapy}

The success of ibrutinib monotherapy has prompted several combination trials, in which ibrutinib is administered with therapeutic monoclonal antibodies (eg, rituximab and ofatumumab), chemotherapy (eg, FCR and BR), and select targeted immune therapies (eg, lenalidomide). Of the studies presented to date, combination approaches have generally yielded improved ORRs in part through earlier resolution of persistent lymphocytosis. 
However, none of these studies were randomized, and it is unclear if combination therapy will affect response duration versus single-agent therapy.

\section{How Should Patients With Ibrutinib Resistance Be Managed?}

In most cases of CLL relapse and some cases of Richter's transformation, mutations of either the BTK gene at the C481 residue, where the drug irreversibly binds, or the PLCGY2 gene, the immediate downstream target of BTK, are found ${ }^{19}$. Cessation of ibrutinib therapy in relapsing patients can sometimes result in rapid tumor progression, particularly for patients with previously heavily treated CLL or Richter's transformation.

Therefore, current practice is not to discontinue ibrutinib therapy until immediately before the next treatment is initiated. The experience so far showed that patients with CLL with ibrutinib progression have been responsive to other CLL therapies. In contrast, the outcome of Richter's transformation after ibrutinib is poor; responses to other therapies, including investigational agents, have been observed but have often been of brief duration. Allogeneic stem-cell transplantation for Richter's transformation of CLL at any time point in the disease should be strongly considered.

\section{References}

1. Byrd JC, Jones JJ, Woyach $\mathrm{J}$, et al. Entering the era of targeted therapy for chronic lymphocytic leukemia: impact on the practicing clinician. J Clin Oncol. 2014; 32(27):30393047.

2. Stevenson FK, Krysov S, Davies AJ, et al. B-cell receptor signaling in chronic lymphocytic leukemia. Blood. 2011; 118(16):4313-4320.

3. Pierce SK and Liu W. The tipping points in the initiation of B cell signalling: how small changes make big differences. Nat Rev Immunol. 2010; 10(11):767-777.

4. Hashimoto A, Okada $\mathrm{H}$, Jiang $\mathrm{A}$, et al. Involvement of guanosine triphosphatases and phospholipase $\mathrm{C}$-gamma2 in extracellular signal-regulated kinase, c-Jun $\mathrm{NH}$-terminal kinase, and p38 mitogen-activated protein kinase activation by the $\mathrm{B}$ cell antigen receptor. J Exp Med. 1998; 188(7):1287-1295.

5. Ingley E. Src family kinases: Regulation of their activities, levels and identification of new pathways. Biochim Biophys Acta. 2008; 1784(1):56-65.

6. Mockridge $\mathrm{Cl}$, Potter KN, Wheatley I, Neville LA, et al. Reversible anergy of slgM-mediated signaling in the two subsets of CLL defined by $\mathrm{VH}$-gene mutational status. Blood. 2007; 109(10):4424-4431.

7. Petlickovski A, Laurenti L, Li X, et al. Sustained signaling through the B-cell receptor induces Mcl-1 and promotes survival of chronic lymphocytic leukemia B cells. Blood. 2005; 105(12):4820-4827. 
8. Wiestner A, Rosenwald A, Barry TS, et al. ZAP-70 expression identifies a chronic lymphocytic leukemia subtype with unmutated immunoglobulin genes, inferior clinical outcome, and distinct gene expression profile. Blood. 2003; 101(12):4944-4951.

9. Chen L, Huynh L, Apgar J, et al. ZAP-70 enhances IgM signaling independent of its kinase activity in chronic lymphocytic leukemia. Blood. 2008; 111(5):2685-2692.

10. Wickremasinghe RG, Prentice AG, Steele AJ. Aberrantly activated anti-apoptotic signaling mechanisms in chronic lymphocytic leukaemia cells: clues to the identification of novel therapeutic targets. Br J Haematol. 2011; 153(5):545-556.

11. Poggi A, Prevosto C, Catellani S, Rocco I, Garuti A, Zocchi MR. Engagement of CD31 delivers an activating signal that contributes to the survival of chronic lymphocytic leukaemia cells. Br J Haematol. 2010; 151(3):252-264.

12. Advani RH, Buggy JJ, Sharman JP, et al. Bruton tyrosine kinase inhibitor ibrutinib (PCI$32765)$ has significant activity in patients with relapsed/refractory B-cell malignancies. J Clin Oncol. 2013; 31(1):88-94.

13. Byrd JC, Furman RR, Coutre SE, et al. Targeting BTK with ibrutinib in relapsed chronic lymphocytic leukemia. N Engl J Med. 2013; 369(1):32-42.

14. Liu J, Fitzgerald ME, Berndt MC, et al. Bruton tyrosine kinase is essential for botrocetin/VWF induced signaling and GPIb-dependent thrombus formation in vivo. Blood. 2006; 108:2596-2603.

15. Brown JR, Furman RR, Flinn I, et al. Final results of a phase I study of idelalisib (GS1101) a selective inhibitor of $\mathrm{PI} 3 K_{\text {_, }}$, in patients with relapsed or refractory CLL (abstract). J Clin Oncol 2013; 31(15) abstract 7003.

16. Barrientos JC, Furman RR, Leonard J, et al. Update on a phase I study of the selective PI3K_ inhibitor idelalisib (GS-1101) in combination with rituximab and/or bendamustine in patients with relapsed or refractory CLL (abstract). J Clin Oncol. 2013; 31 (15) abstract 7017.

17. Patel M, Kahl B, Horwitz S, et al. Preliminary safety and efficacy of IPI-145, a potent inhibitor of phosphoinositide-3-kinase- $\delta, \gamma$, in patients with relapsed/refractory CLL. ASCO Meeting Abstracts. 2013; 30:7070.

18. Seymour JF, Davids MS, Pagel JM, et al. Updated results of a phase I first-in-human study of the BCL-2 inhibitor ABT-199 (GDC-0199) in patients with relapsed/refractory (R/R) chronic lymphocytic leukemia (CLL) (abstract). J Clin Oncol. 2013; 31(15) Abstract 7018.

19. Hallek M, Fischer K, Fingerle-Rowson G, et al. International Group of Investigators; German Chronic Lymphocytic Leukaemia Study Group. Addition of rituximab to fludarabine and cyclophosphamide in patients with chronic lymphocytic leukaemia: a randomised, open label, phase 3 trial. Lancet. 2010; 376(9747):1164-1174. 